\title{
Implementación de un entorno de aprendizaje virtual integrando herramientas de $e$-learning y CMS
}

\section{Resumen}

La investigación tiene como objetivo describir las diferentes plataformas de e-learning, así como las soluciones de administradores de contenido (CMS) existentes en el mercado. Asimismo, se implementa un portal virtual integrando Moodle y Joomla a través de una extensión llamada Joomdle. Con este tipo de soluciones se podrá proporcionar al usuario mayor facilidad al momento de llevar cursos virtuales.

\section{Palabras clave}

Plataformas virtuales, e-learning, Moodle, Joomla, CMS, Joomdle.

\author{
Carlos Antonio Aguirre-Ayala' \\ Docente Utec \\ carlos.aguirre@utec.edu.sv \\ Marvin Elenilson Hernández-Montoya ${ }^{2}$ \\ Docente Utec \\ marvin.hernandez@utec.edu.sv
}

Recibido: 29/03/2016 - Aprobado: 28/04/2016 


\section{Introducción}

La educación es un pilar fundamental para las sociedades en vías de desarrollo, ya que por medio del conocimiento se obtienen los escenarios reales que se convierten en la fuerza impulsora para la generación de innovación que, a su vez, tiene su origen en la investigación. Gracias a los aportes tecnológicos, las barreras que reducen las posibilidades de acceder a cursos de capacitación y carreras profesionales ahora son menores, ya que los medios en la administración de contenido permiten brindar una serie de servicios enfocados en la oferta de programas curriculares que permiten incrementar el conocimiento y desarrollar competencias en ambientes flexibles de aprendizaje, puesto que no dependen de horarios ni de ubicación física.

Para esta investigación se busca estudiar la plataforma de e-learning Moodle y el CMS Joomla, integrando la potencialidad de ambos a través de una extensión llamada Joomdle. Muchas instituciones de educación utilizan Moodle para impartir sus cursos virtuales, el cual ofrece una diversidad de opciones que hacen que un curso en esta plataforma sea dinámico y amigable; sin embargo, presenta algunas dificultades por su diseño plano y no muy vistoso, con su integración con Joomla, que es un CMS muy reconocido por su vistosidad y potencia en el manejo de portales corporativos. Esto significará que, al fusionar ambas plataformas, Moodle tendrá todo su potencial en el manejo de cursos virtuales y, a su vez, presentará un entorno amigable y profesional.

Con este trabajo de investigación se busca lo siguiente: a) Identificar las características técnicas de Moodle y Joomla para su instalación. b) Instalar y configurar Moodle y Joomla. c) Instalar y configurar Joomdle. d) Implementación del portal virtual.

Con la implementación del portal en Joomla y Moodle el usuario del aula virtual podrá lograr lo siguiente:

- Hacer el sitio web responsivo para cualquier plataforma (dispositivos móviles, PC, laptop, entre otros)

- Tendrá una sola clave de ingreso y facilidad para recuperar su clave si se le olvida.
- Sitio web flexible, liviano y vistoso.

- Podrá visualizar toda la información del portal institucional, dado que ambas plataformas estarán integradas.

\section{La educación del futuro}

La revolución tecnológica predispone cambios en todas las esferas de la vida social, entre ellas, en la (ESFERA) de la educación. Según la Cumbre Mundial para la Innovación en Educación (Wise, por sus siglas en inglés), en los próximos 15 años los colegios se convertirán en entornos interactivos y el prototipo de escuela, tal como se conoce hoy, cambiará. La clase magistral desaparecerá y el profesor se convertirá en guía del alumno. Wise considera que el aprendizaje será personalizado, permanente y más caro. Según la investigación publicada en las redes, internet será la principal fuente y el inglés la lengua mayoritaria, y los sistemas educativos de todo el mundo sufrirán grandes modificaciones por la revolución tecnológica (Wise, 2015).

\section{E-learning}

El concepto de e-learning es una modalidad de enseñanzaaprendizaje que consiste en el diseño, puesta en práctica y evaluación de un curso o plan formativo desarrollado a través de redes de ordenadores; y puede definirse como una educación o formación ofrecida a individuos que están geográficamente dispersos o separados o que interactúan en tiempos diferidos, empleando los recursos informáticos y de telecomunicaciones (Moreira, 2015).

\section{Las plataformas de e-learning}

Es un espacio virtual de aprendizaje orientado a facilitar la experiencia de capacitación a distancia, tanto para empresas como para instituciones educativas. Este sistema permite la creación de aulas virtuales; en ellas se produce la interacción entre tutores y alumnos, y entre los mismos alumnos; en este se realizan las evaluaciones, el intercambio de archivos, la participación en foros, chats y otras herramientas adicionales (E-ABC, 2015).

Las siguientes son las características de una plataforma de e-learning (Clarenc, 2013). 

a) Interactividad
e) Usabilidad
b) Flexibilidad
f) Ubicuidad
c) Escalabilidad
g) Persuabilidad
d) Estandarización
h) Accesibilidad

Algunas de las plataformas de e-learning más utilizadas son las siguientes:
a) Dokeos
f) E-doceo
b) Ilias
g) WebCT
c) Atutor
h) Blackboard
d) Moodle
i) Skillfactory
e) Claroline

\section{Sistemas de administración de contenido (CMS)}

Es una aplicación informática utilizada para la creación, edición, administración y publicación de información de una forma fácil, organizada y que no requiere de conocimientos especializados sobre edición digital. Generalmente la interfaz administrativa es sencilla y amigable, permitiendo que cualquier persona pueda administrar sus propios contenidos web sin necesidad de poseer conocimientos técnicos o lenguaje HTML (UNAM, 2015)

Principales ventajas de los CMS (Unam, 2015):

- Organización del sitio web

- Publicación de contenidos

- Escalabilidad e implementación de nuevas funcionalidades

- Administración de usuarios

- Diseño y aspecto estético del sitio

- Navegación y menú

- Administrador de imágenes

- Disposición de módulos modificable

- Automatización en la publicación

- Archivo e historial
- Formatos de lectura

- Comentarios

CMS más utilizados:

- Drupal

- WordPress

- Joomla

\section{Método}

La investigación será descriptivo-experimental, se realizará una descripción de las características de los elementos involucrados en la investigación, tales como Joomla, Moodle y Joomdle.

\section{Sujetos de estudio}

Plataformas Moodle y Joomla.

\section{Instrumentos que se utilizarán para recolección de datos}

Análisis documental por medio del cual se buscará obtener información valiosa que ayude a la implementación de un entorno de aprendizaje virtual integrando herramientas de e-learning y CMS.

\section{Procedimientos}

Lo primero que se realizó fue investigar, en diferentes medios, información relevante sobre los sujetos de estudio, llámense Moodle y Joomla, luego se identificaron características, requerimientos de instalación y todos aquellos aspectos que ayudaran a montar el portal colaborativo. Como siguiente paso se compró el hosting y se procedió a instalar Joomla y Moodle. Después de realizada la instalación, se verificó que funcionaran correctamente y se procedió a instalar y configurar Joomdle.

Finalizando todo el proceso con la personalización del portal, integrando ambas plataformas Joomla y Moodle en una sola. 


\section{Resultados}

\section{Instalación de Joomdle}

Para descargar los archivos de instalación de Joomdle, debe visitarse http://sourceforge.net/projects/moodle-joomla/files/, que es el sitio oficial del proyecto.

\begin{tabular}{|c|c|c|c|c|}
\hline Home & & & & \\
\hline Name * & Modified * & Size $*$ & Downloads / Week * & \\
\hline joomdle_v1.0.0-for-joomla30-UNZIP-... & 2014-12-16 & $914.7 \mathrm{kB}$ & 164 & i \\
\hline joomdle-0.95-for-joomla30.zip & 2014-02-24 & $973.8 \mathrm{kB}$ & 1,0 & (i) \\
\hline joomdle-0.95-for-joomla25.zip & 2014-02-24 & 969.2 kB & $4 \perp$ & i \\
\hline joomdle-0.94-for-joomla30.zip & 2013-11-22 & $976.6 \mathrm{kB}$ & 1. & i \\
\hline joomdle-0.94-for-joomla25.zip & 2013-11-22 & $977.7 \mathrm{kB}$ & $1 \square$ & i \\
\hline joomdle-0.93-for-joomla30.zip & 2013-10-29 & $970.4 \mathrm{kB}$ & $1 \square$ & i \\
\hline joomdle-0.93-for-joomla25.zip & 2013-10-29 & $968.3 \mathrm{kB}$ & $1 \square$ & i \\
\hline
\end{tabular}

Figura 1. Sitio de descarga de Joomdle

Fuente: Elaboración propia.

- Se descomprime el archivo descargado y luego se copian las carpetas auth_joomdle y enrol_joomdle que se encuentran en la carpeta que se descomprimió; y se copian dentro de las carpetas auth y enrol que están dentro del directorio de Moodle.

\begin{tabular}{|c|c|c|c|}
\hline Nombre & Fecha de modifica... & Tipo & Tamaño \\
\hline Ds auth_joomdle & $04 / 06 / 201509: 55 \ldots$ & Carpeta de archivos & \\
\hline DS enrol_joomdle & 04/06/2015 09:55 ... & Carpeta de archivos & \\
\hline Thath_joomdle.zip & 27/02/2015 11:56 a... & Carpeta comprimi... & $77 \mathrm{~KB}$ \\
\hline It enrol_joomdle.zip & 27/02/2015 11:56 a... & Carpeta comprimi... & $20 \mathrm{~KB}$ \\
\hline
\end{tabular}

Figura 2. Sitio de descarga de Joomdle

Fuente: Elaboración propia. 
- Se abre Moodle, como administrador, desde el URL http://localhost/moodle/admin/

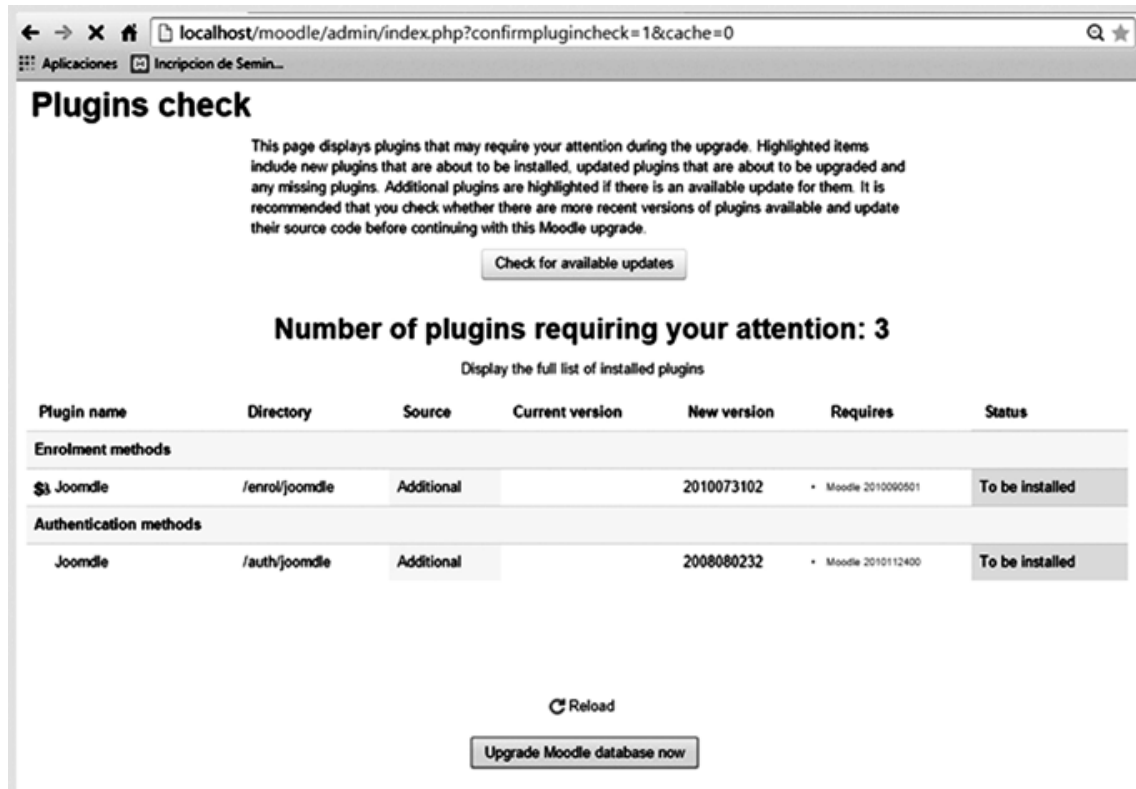

Figura 3. Actualizar base de datos Joomdle

Fuente: Elaboración propia.

- Se procede a la activación de la extensión Joomdle en Moodle.

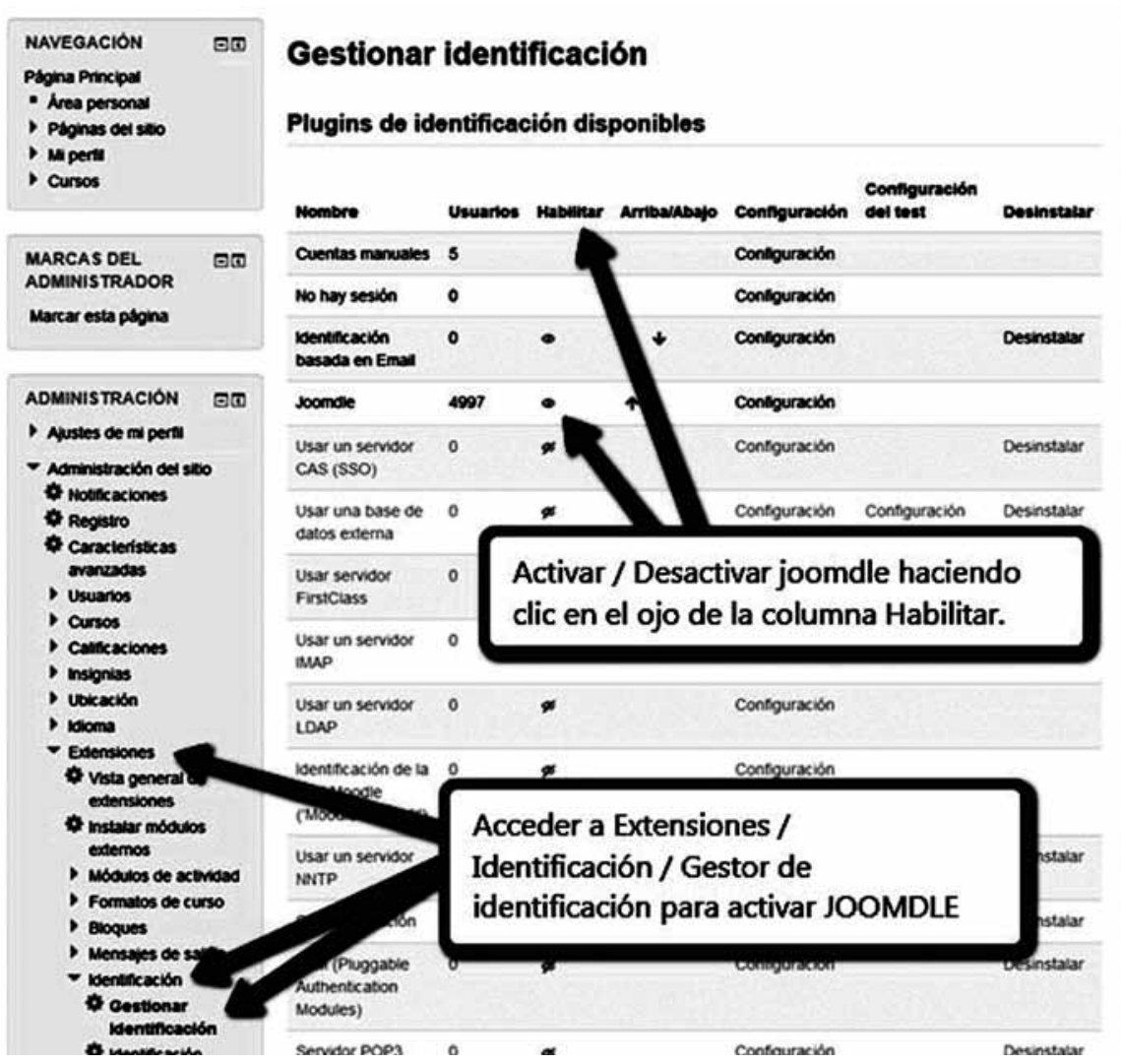

Figura 4. Actualizar base de datos Joomdle

Fuente: Elaboración propia. 
- Se habilitan los servicios web correspondientes.

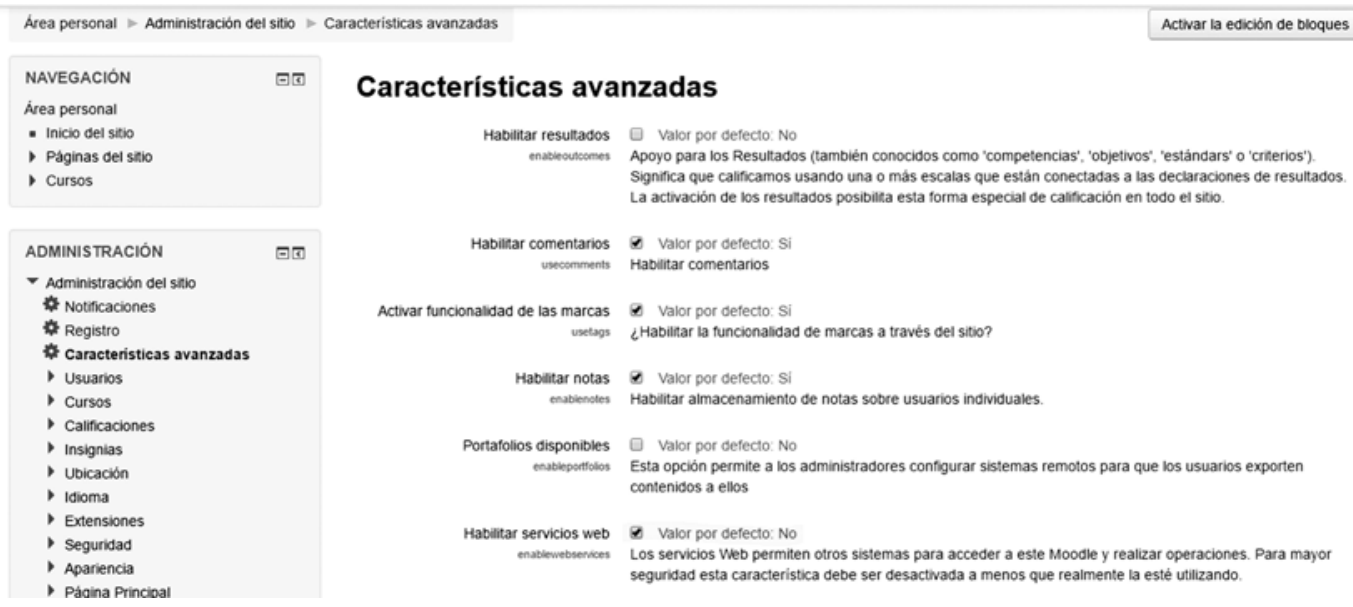

Figura 5. Actualización de servicios web

Fuente: Elaboración propia.

- Se activan protocolos XML-RPC en Moodle; Joomdle usa XML-RPC para conectar con Moodle. Se debe seleccionar Habilitar protocolos, de la lista, y activar XML-RPC. Es posible que algunos servidores además requieran habilitar el protocolo SOAP. Para la configuración debe seguir la ruta Administración del sitio- Extensiones-Servicios web-Administrar protocolos.

\begin{tabular}{|c|c|c|c|c|c|}
\hline \multicolumn{5}{|c|}{ Área personal $>$ Administración del sitio $>$ Extensiones $>$ Servicios Web $>$ Administrar protocolos } & Activar la edición de bioques \\
\hline \multirow{3}{*}{$\begin{array}{l}\text { NAVEGACIÓN } \\
\text { Área personal } \\
\text { - Inicio del sitio } \\
\text { Páginas del sitio } \\
\text { - Cursos }\end{array}$} & \multirow[t]{3}{*}{ घब } & \multirow{2}{*}{\multicolumn{4}{|c|}{$\begin{array}{l}\text { Administrar protocolos } \\
\text { Protocolos activos de servicio web }\end{array}$}} \\
\hline & & & & & \\
\hline & & Protocolo & Versión & Habilitar & Configuración \\
\hline \multirow{6}{*}{$\begin{array}{l}\text { ADMINIS TRACIÓN } \\
\text { Administracion del stilo } \\
\text { Notincaciones } \\
\text { Registro } \\
\text { Caracteristicas avanzadas } \\
\text { Usuarios } \\
\text { Cursos } \\
\text { Califcaciones } \\
\text { Insignias } \\
\text { - Ubicación } \\
\text { - lalioma }\end{array}$} & \multirow[t]{6}{*}{ घब } & Protocolo AMF & 2015111600 & $\%$ & \\
\hline & & Protocolo REST & 2015111600 & \% & \\
\hline & & Protocolo SOAP & 2015111600 & \% & \\
\hline & & Protocolo XML-RPC & 2015111600 & - & \\
\hline & & \multicolumn{4}{|c|}{ Por razones de seguridad, solo se habilitaran los protocolos que vayan a ser utilizados. } \\
\hline & & Documentación de servicios web & $\begin{array}{l}\text { Maran los prolocolos que : } \\
\text { Habilitar la la auto-genera } \\
\text { documentación en su p. } \\
\text { protocolos habilitados. }\end{array}$ & ión de serv & $\begin{array}{l}\text { usuario puede tener acceso a su propia } \\
\text { olo se muestra la documentación de los }\end{array}$ \\
\hline
\end{tabular}

Figura 6. Protocolo XML-RPC

Fuente: Elaboración propia. 
- Se comprueban los permisos de usuario para conectar con Joomdle, para ello se sigue la ruta Administración del sitioUsuarios-Permisos-Definir roles.

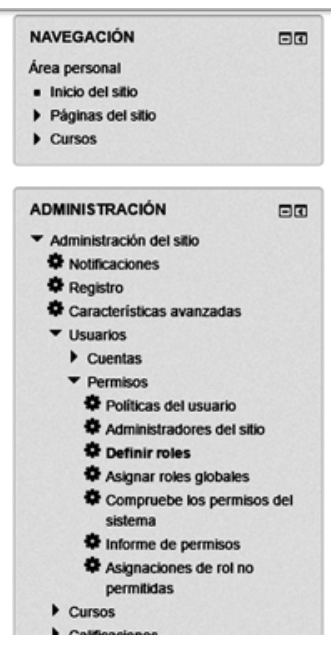

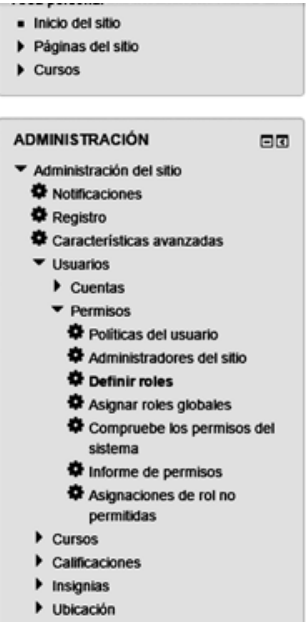

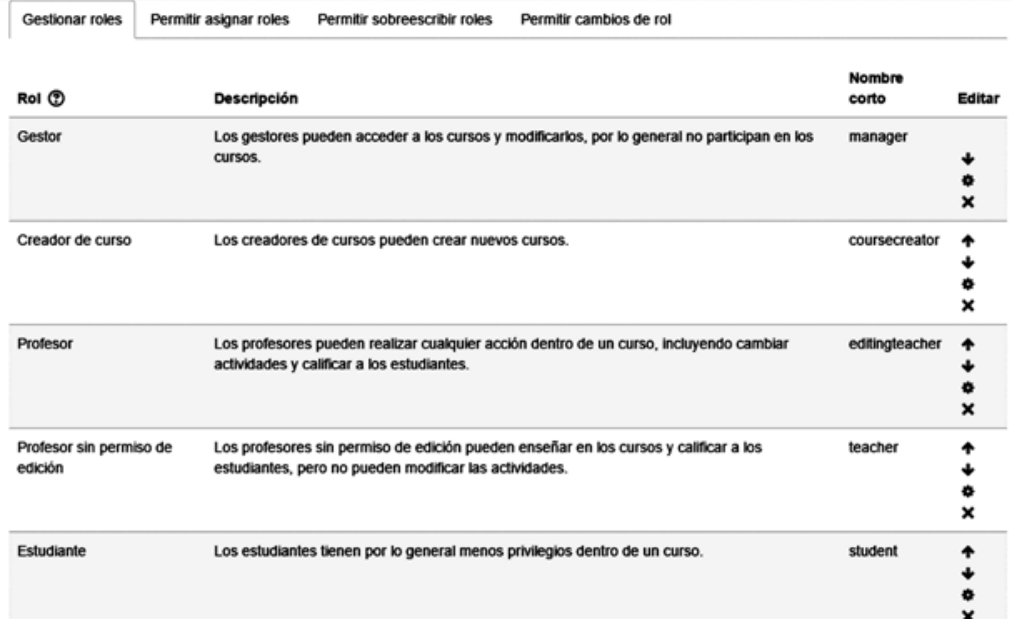

Figura 7. Permisos de usuario

Fuente: Elaboración propia

\section{Editando el rol 'webservice'๑}

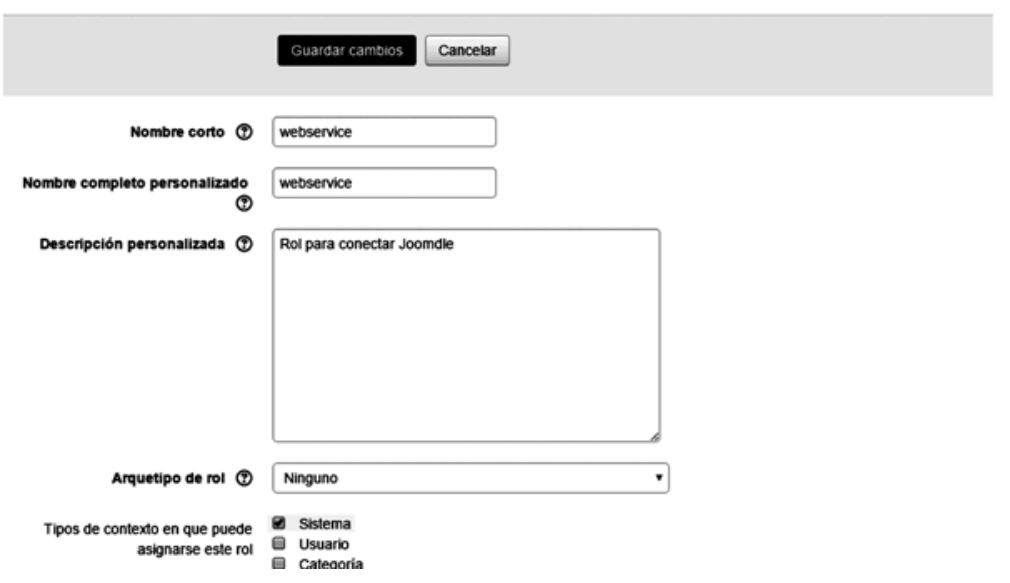

Figura 8. Configuración de permisos

Fuente: Elaboración propia. 
- Se habilitan las capacidades del rol para el usuario:

- Usar protocolo XML-RPC webservice/xmlrpc. Use: Permitir.

- Usar protocolo SOAP webservice/soap. Use: Permitir.

- Ver debatesmod/fórum viewdiscussion. Use: Permitir.

\begin{tabular}{|c|c|}
\hline $\begin{array}{l}\text { Usar protocolo AMF } \\
\text { websenvioe/amfuse }\end{array}$ & $\stackrel{\square}{\square}$ \\
\hline \multicolumn{2}{|l|}{ Servicio Web: Protocolo REST } \\
\hline $\begin{array}{l}\text { Usar protocolo REST } \\
\text { websenticetrestuse }\end{array}$ & $\begin{array}{c}\square \\
\text { Permitir }\end{array}$ \\
\hline \multicolumn{2}{|l|}{ Servicio Web: Protocolo SOAP } \\
\hline $\begin{array}{l}\text { Usar protocolo SOAP } \\
\text { websenkce/soapuse }\end{array}$ & Permitir \\
\hline \multicolumn{2}{|l|}{ Servicio Web: Protocolo XML-RPC } \\
\hline $\begin{array}{l}\text { Usar protocolo XML-RPC } \\
\text { websenko/monumpeuse }\end{array}$ & Permitir \\
\hline \multicolumn{2}{|l|}{ Exportar Libro a IMS CP } \\
\hline $\begin{array}{l}\text { Exportar libro como paquete de contenido IMS } \\
\text { booktoovexportimspiespot }\end{array}$ & $\begin{array}{c}\square \\
\text { Permitir }\end{array}$ \\
\hline
\end{tabular}

Figura 9. Capacidades del rol para el usuario

Fuente: Elaboración propia.

- Se añade al nuevo rol creado, Servicios web, el usuario al que se le asignará Joomdle. Se sigue la ruta Administración del sitio-Usuarios-Permisos-Asignar roles globales.

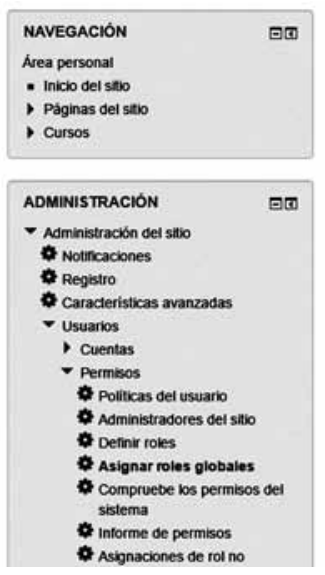

\section{Asignar rol 'webservice' en Sistema $\odot$}

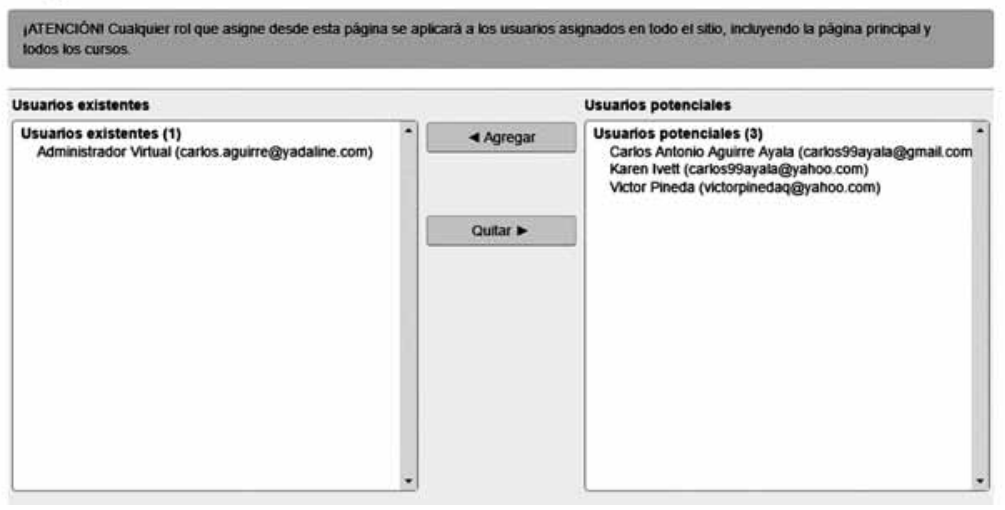

Figura 10. Usuario asociado a Joomdle

Fuente: Elaboración propia. 
- Se agrega un nuevo servicio para Joomdle. Se sigue la ruta Administración del sitio- Extensiones-Servicios web-Servicios externos.

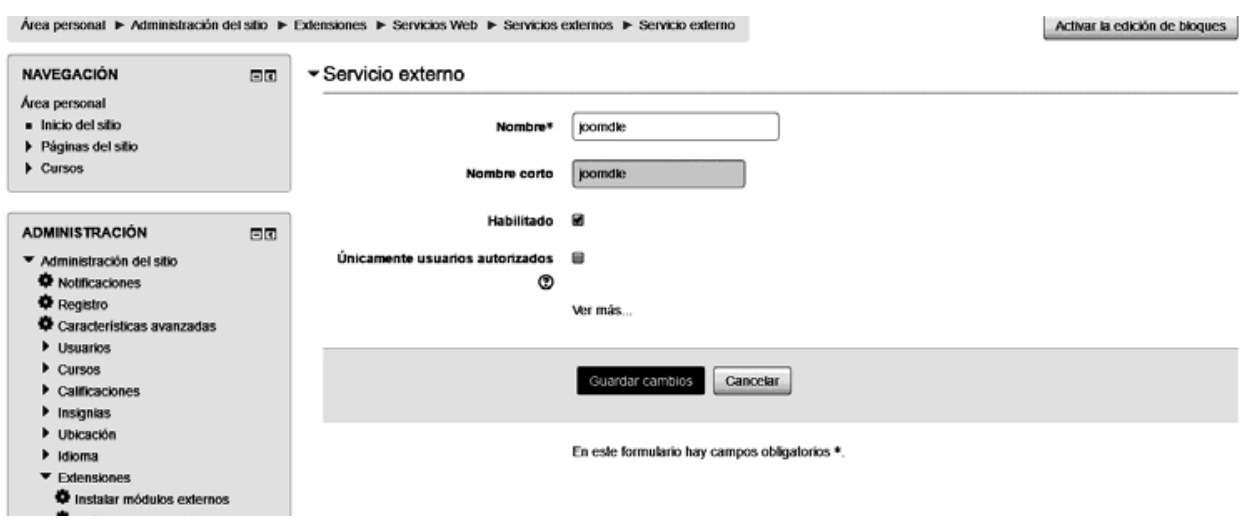

Figura 11. Usuario asociado a Joomdle

Fuente: Elaboración propia.

- Se agregan funciones al servicio externo de Joomdle en Moodle. Se sigue la ruta Administración del sitio-ExtensionesServicios web Servicios externos-Funciones- Agregar funciones.

\section{por el Mundo}

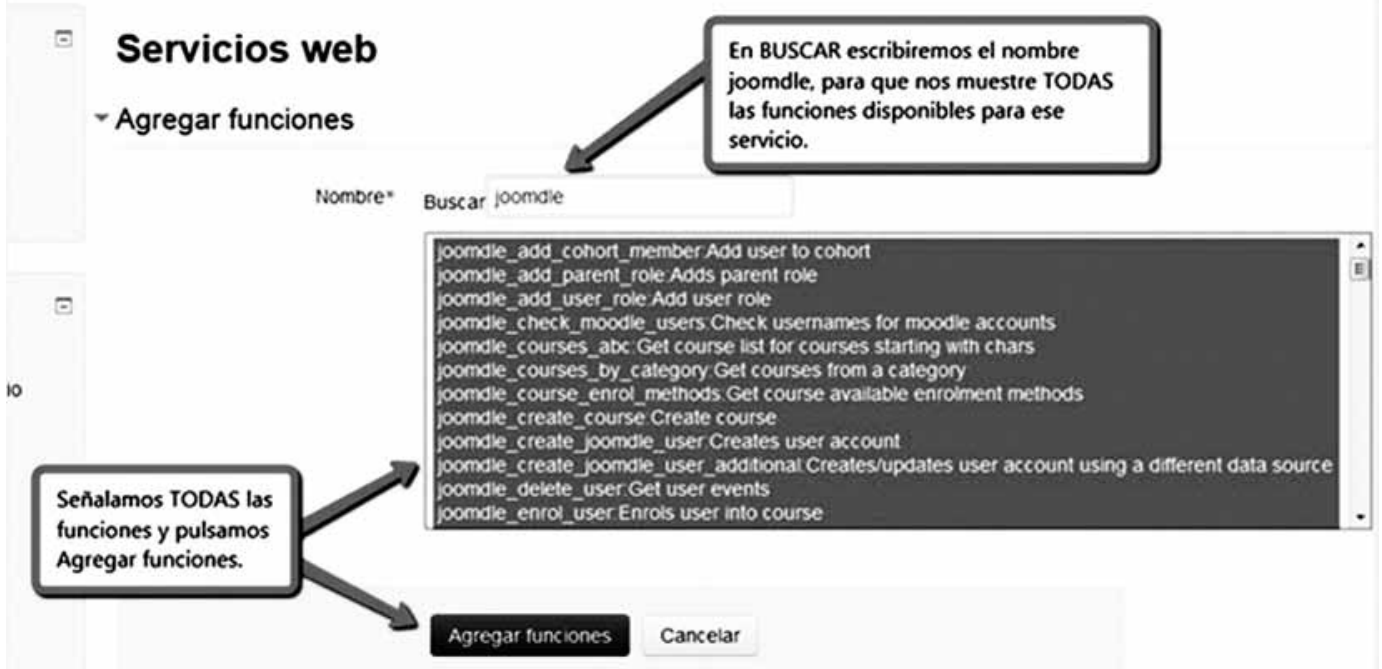

Figura 12. Agregar funciones al servicio externo Joomdle

Fuente: Elaboración propia. 
- Se procede a crear el token de Moodle para Joomdle. Se sigue la ruta Administración del sitio-Extensiones-Servicios webAdministrar tokens-Agregar.

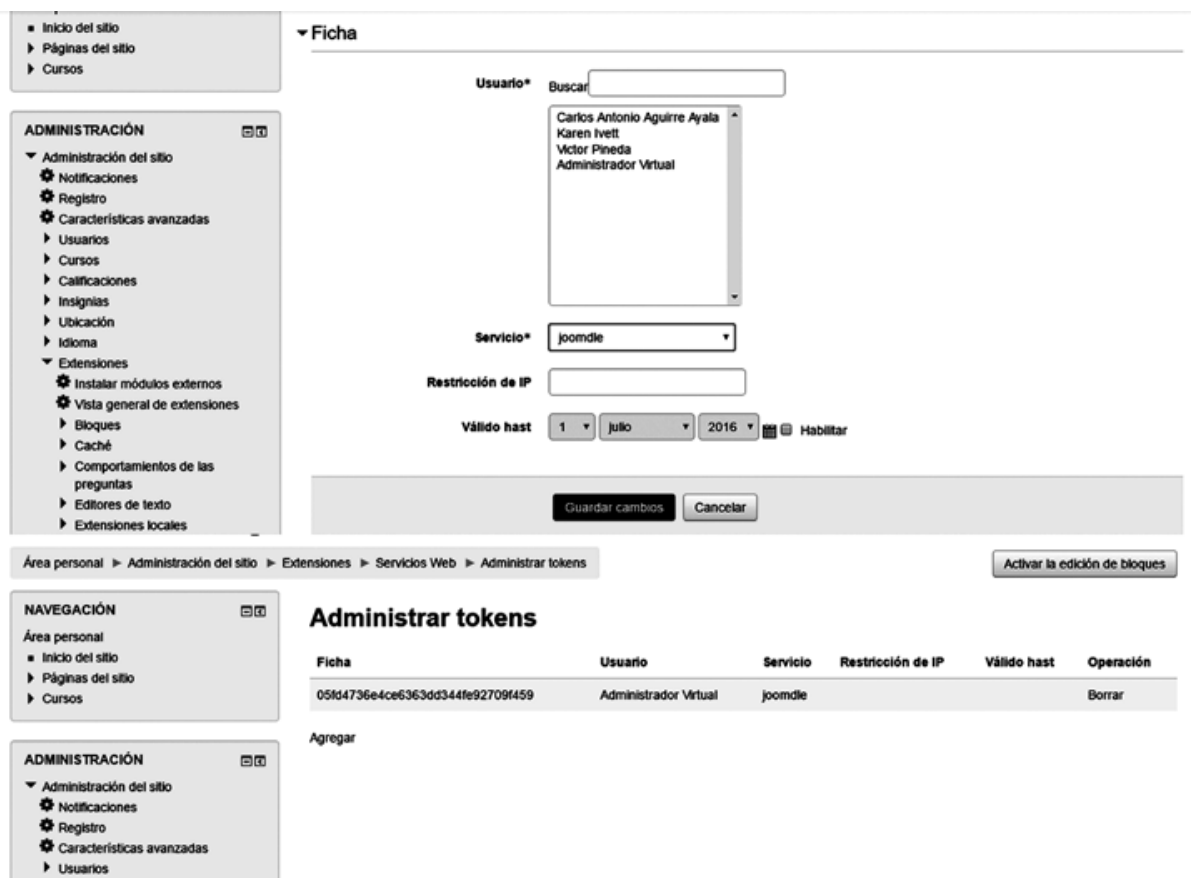

Figura 13. Agregar funciones al servicio externo Joomdle

Fuente: Elaboración propia.

- Se gestiona identificación en Moodle y se asigna URL de Joomla. Para ello se sigue la ruta Administración del sitioExtensiones-Identificación-Gestionar identificación, se da clic en Configuración de Joomdle y luego se escribe el URL en el que está instalado Joomla.

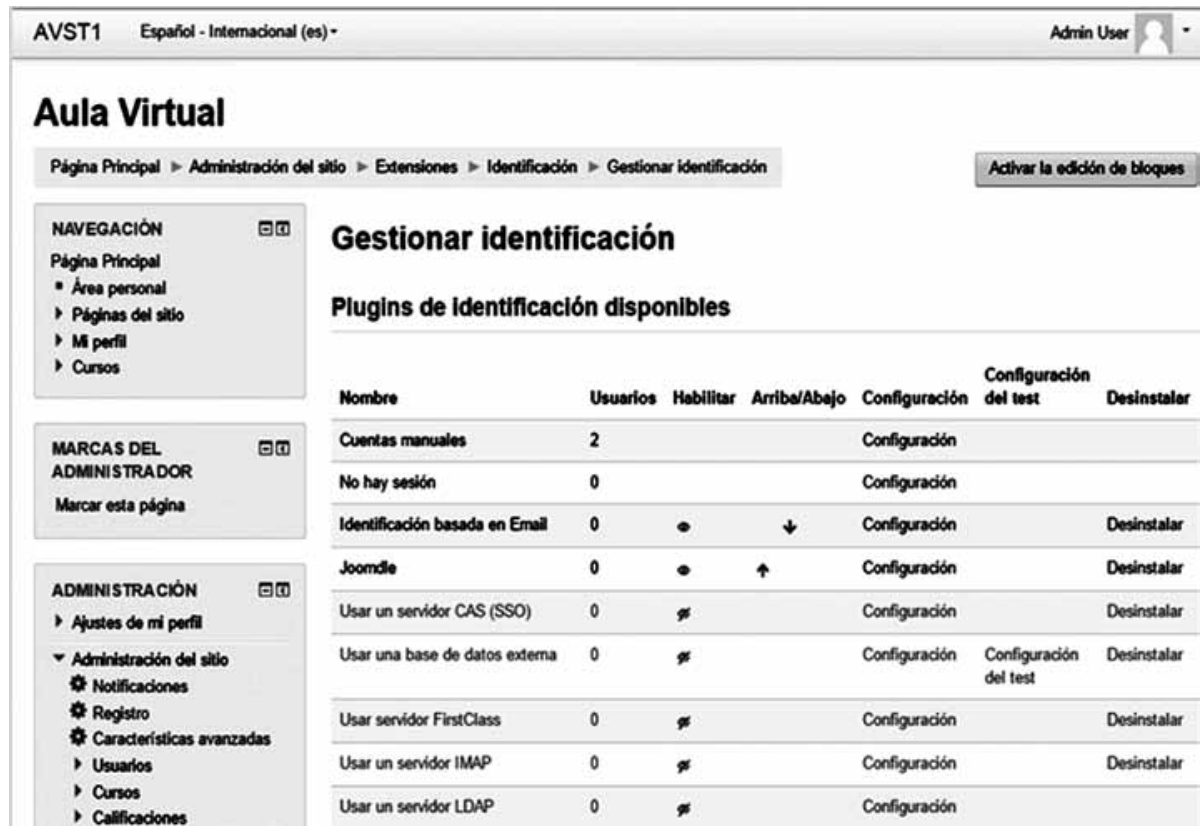

Figura 14. Gestionar identificación de Moodle

Fuente: Elaboración propia. 


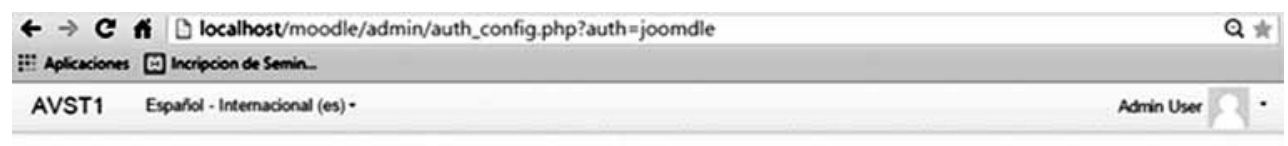

\section{Aula Virtual}

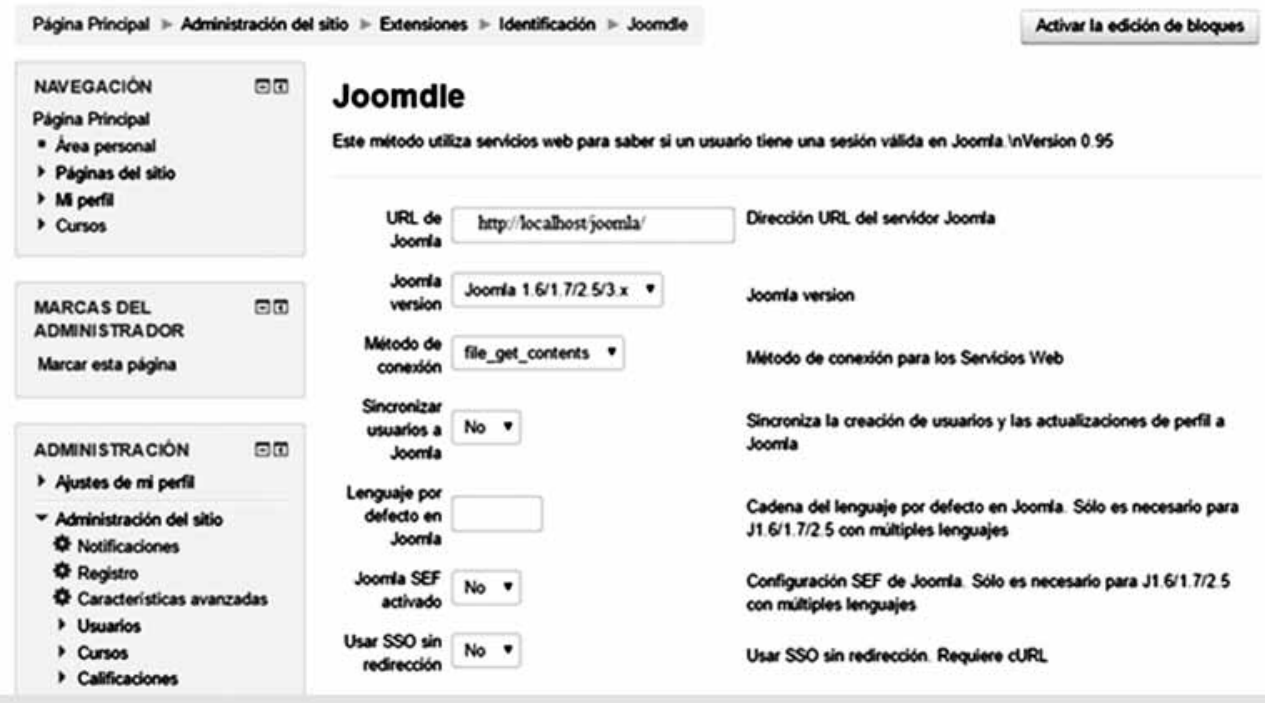

Figura 15. Gestionar identificación de Moodle

Fuente: Elaboración propia.

- Configurando Joomdle en Joomla. Para ello se debe ingresar al URL del módulo de administración donde está instalado Joomla. Para este caso http://localhost/administrator. Especifica su usuario y password.

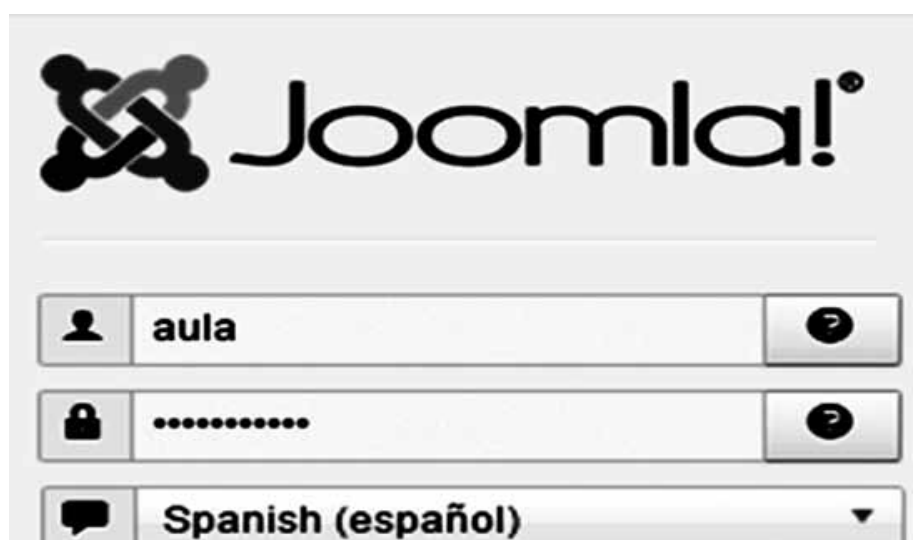

\section{a Log in}

Figura 16. Credenciales de administrador

Fuente: Elaboración propia. 
- Luego se procede a instalar el plugging de Joomdle.

$\leftarrow \rightarrow$ C localhost/joomla/administrator/index.php?option=com_installer

\section{$\$$ Sistema . Usuanos - Menús . Contenido - Componentes . Extensiones . Ayuda , \\ t. Gestor de extensiones: Instalar

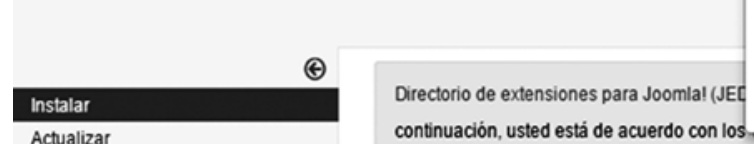

Gestor de módulos

Gestor de plugins

Gestor de plantillas

Gestor de idiomas

lar desde la web desde esta página. Haciendo clic en "Aña

Añadir la pestaña 7nstalar desde la web"

Gestionar

Descubrir

Base de datos

Advertencias

Instalar idiomas

Subir paquete Instalar desde un directorio Instalar desde una URL

Sitios de actualización

Subir e instalar la extensión de Joomla

Archivo del paquete para la extensión Seleccionar archivo Ningún archivo seleccionado

Figura 17. Instalando plugging Joomdle

Fuente: Elaboración propia.

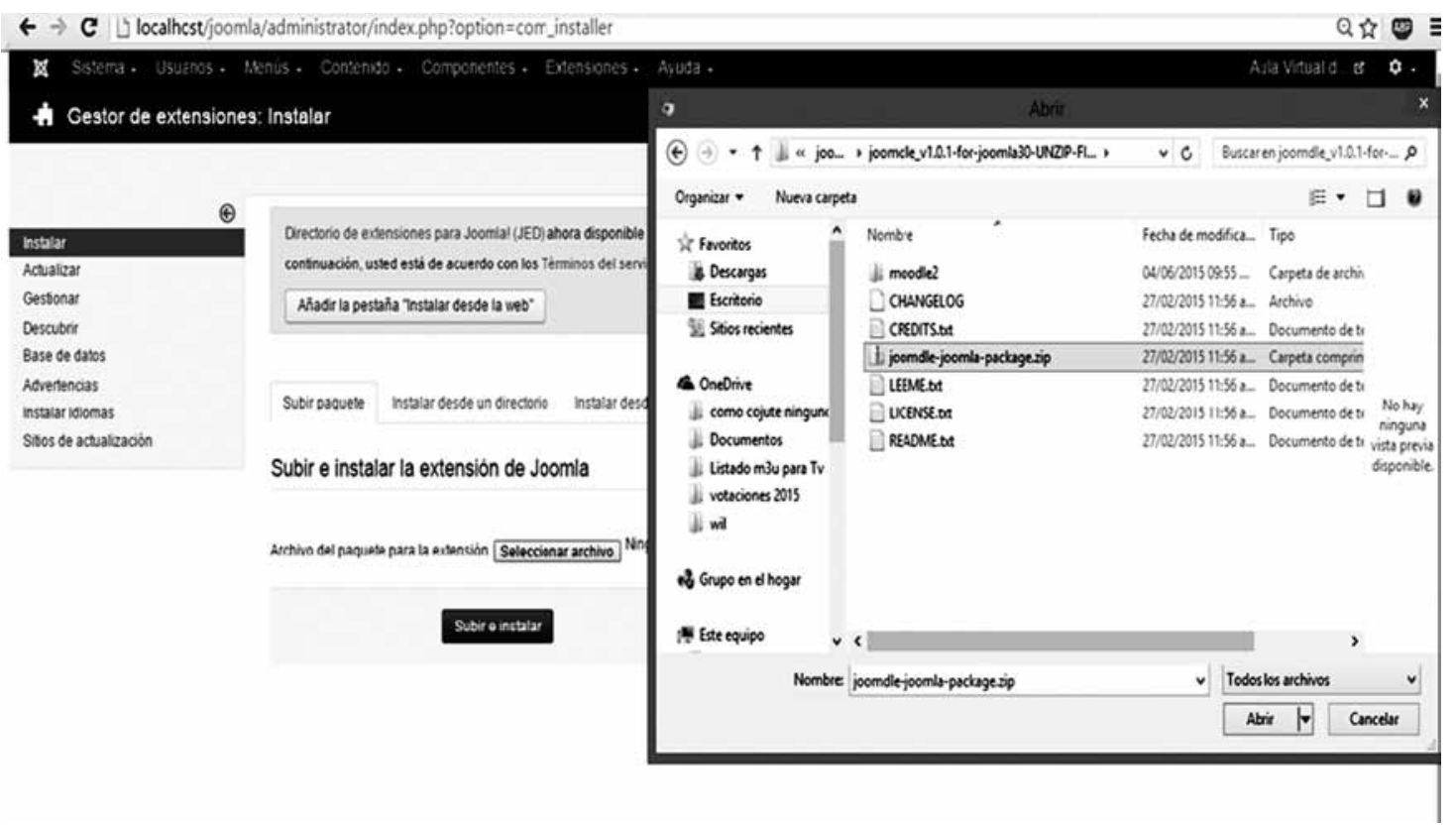

Figura 18. Instalando plugging Joomdle

Fuente: Elaboración propia. 
- Después de la instalación, se procede a configurar el componente Joomdle instalado, para ello se busca la barra de menú del panel de administración la opción Componentes y da clic sobre Joomdle y luego en Panel de control.

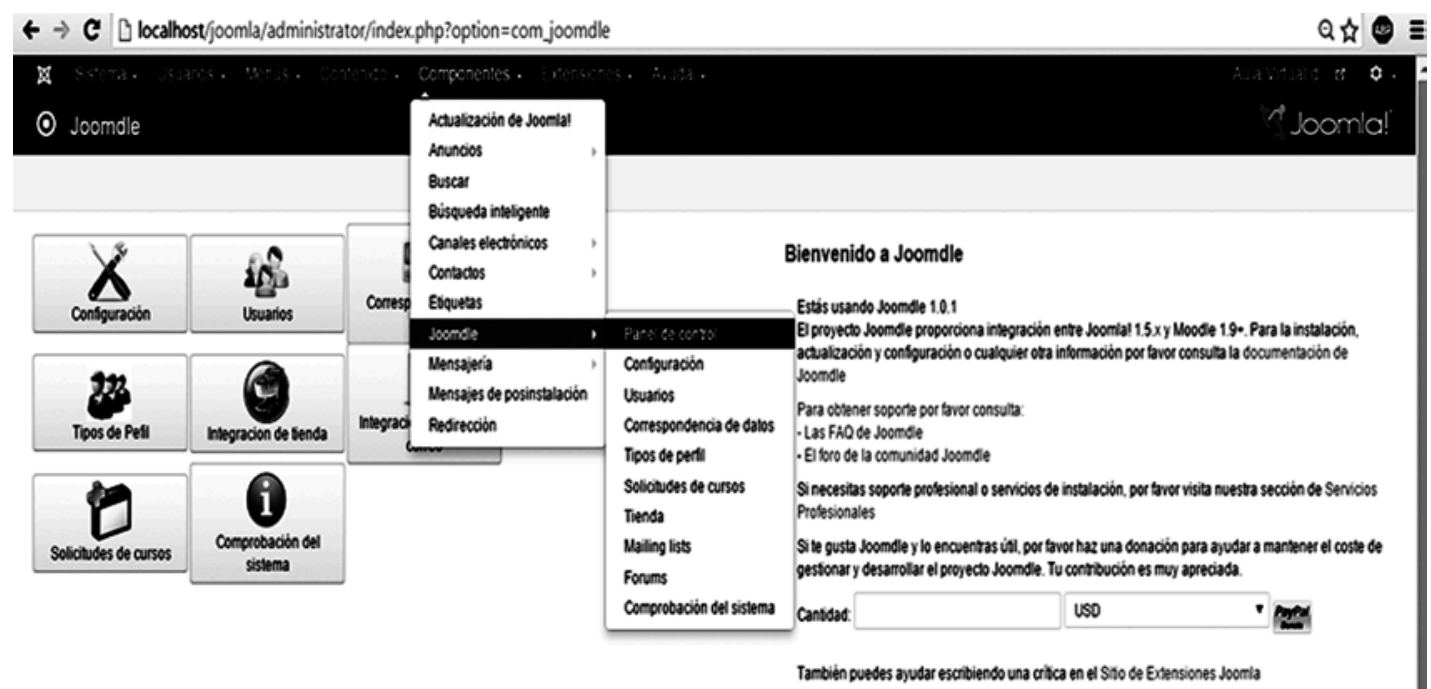

Figura 19. Configuración componente Joomdle

Fuente: Elaboración propia.

- En configuración general, se deberá ingresar el URL donde se tiene instalado moodle, así como el token de autenticación.

\begin{tabular}{|c|c|}
\hline URL de Moodle & http://20.0.0.4/moodle \\
\hline Version de Moodle & Moodle 2.0.x \\
\hline $\begin{array}{l}\text { Token de autenticación de } \\
\text { Moodle } 2.0\end{array}$ & b7c9ef7083cd25b8390a2326b3ee59ac \\
\hline Método de conexión & file_get_contents \\
\hline COM_JOOMDLE_INTERNAL_IP & \\
\hline Usar SSO sin redirección & No \\
\hline $\begin{array}{l}\text { Crear automáticamente } \\
\text { usuarios Moodle }\end{array}$ & \\
\hline $\begin{array}{l}\text { Borrar automáticamente } \\
\text { usuarios Moodle }\end{array}$ & \\
\hline $\begin{array}{l}\text { Auto login de usuarios al } \\
\text { registarse }\end{array}$ & \\
\hline
\end{tabular}

Figura 20. Configuración componente Joomdle

Fuente: Elaboración propia. 
- Pueden realizarse más configuraciones; y dependerá de la necesidad del usuario administrador y lo que él quiera mostrar al usuario final.

- Se finaliza el proceso haciendo la comprobación que Joomla y Moodle se puedan comunicar. En caso de aparecer algún indicador en rojo, habrá que revisar de nuevo todo el proceso para identificar qué es lo que no se hizo bien.

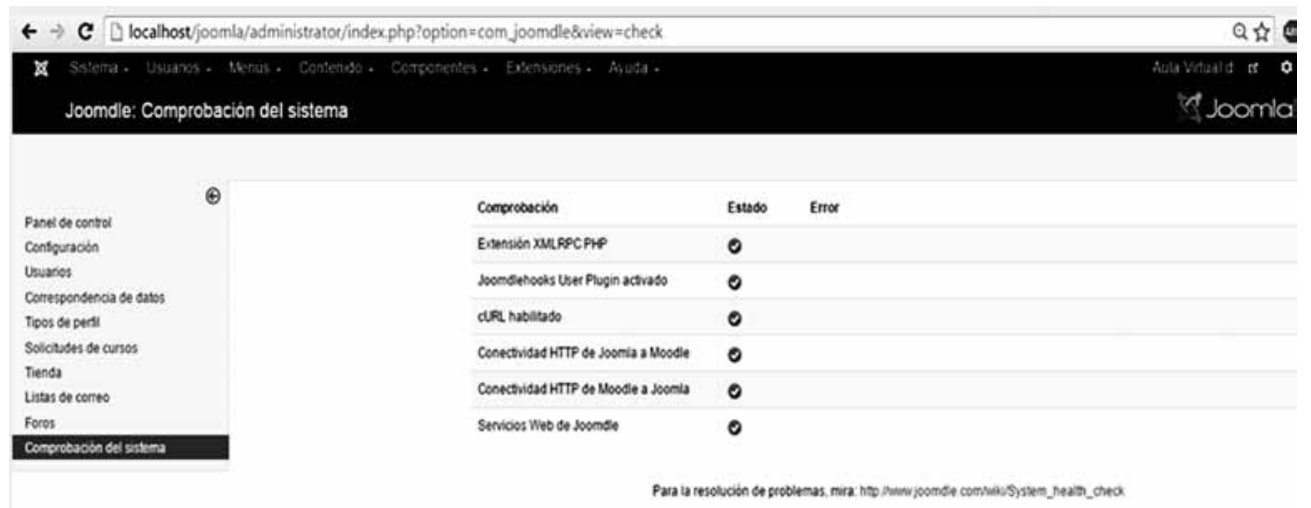

Figura 21. Configuración componente Joomdle

Fuente: Elaboración propia.

- Para mostrar el portal se configurará una entrada del menú principal como Moodle Wrapper. La aplicación se mostrará dentro de un frame, tal y como se observa en la siguiente captura de pantalla. Para visitar el portal web ingrese a http:// www.geniusutec.com

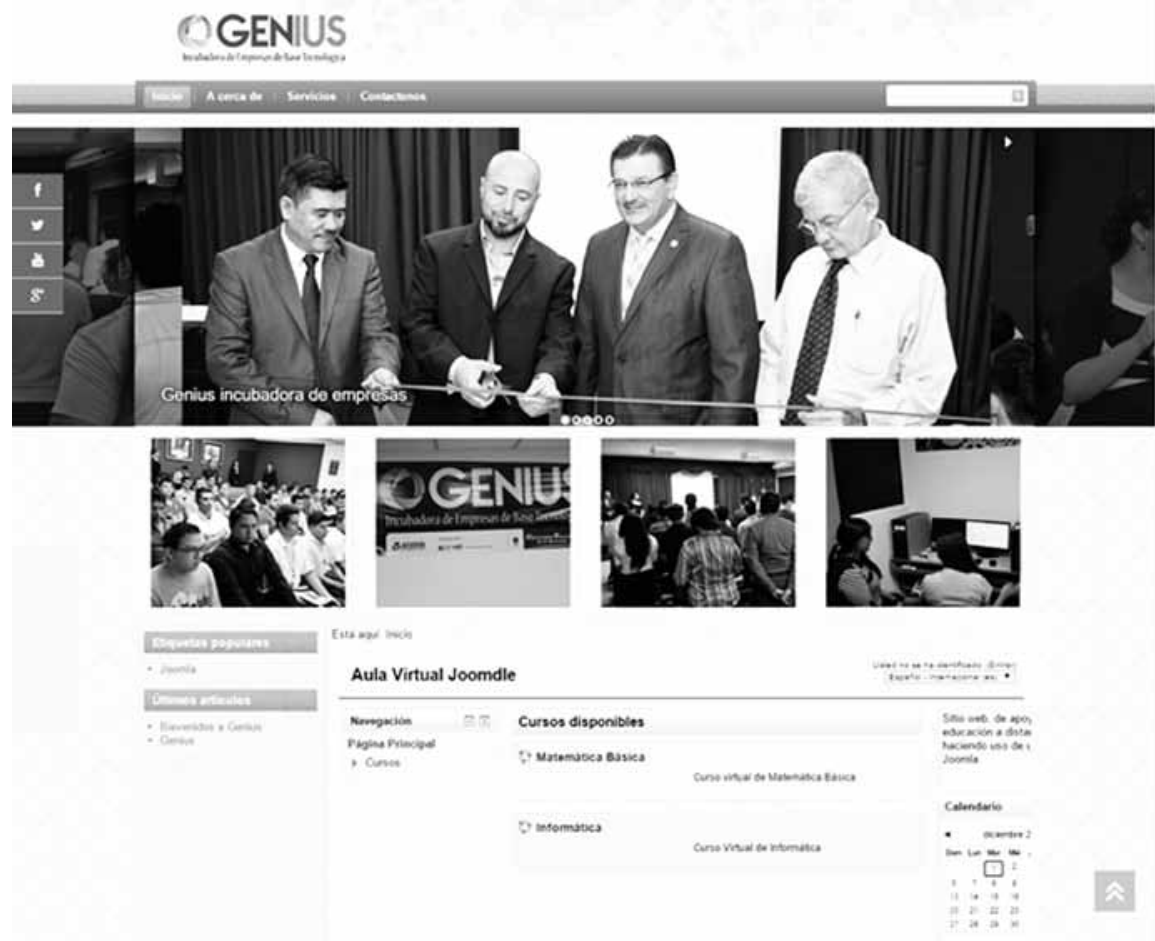

Figura 22. Portal Joomdle

Fuente: Elaboración propia. 


\section{Discusión/conclusión}

Al finalizar la investigación se logra comprobar que la extensión Joomdle permite integrar la plataforma de e-learning Moodle con la de Joomla, con esto se logra obtener una mayor flexibilidad del portal corporativo, así como un portal multiplataforma que podrá visualizarse en cualquier dispositivo o computadora sin perder la trascendencia y usabilidad del sitio web.

Al fusionar ambas plataformas, el usuario podrá hacer uso de Joomla y Moodle sin necesidad de volver a usar credenciales; ya registrado en una plataforma por defecto, estará registrado en la otra. Las sesiones en ambas plataformas están sincronizadas, por lo que la información podrá actualizarse al instante, haciendo del portal un medio de comunicación efectiva.

Joomdle proporciona una agradable experiencia a los usuarios, y para el manejo de clases, cursos, diplomados en modalidad virtual es una excelente herramienta, ya que facilita al estudiante la habilidad de mostrar calificaciones y eventos de tareas que son específicas.

En cuanto a los indicadores del proyecto de investigación, estos han sido alcanzados, pues se integró un portal en Joomla con la plataforma de e-learning Moodle, esto permite que los usuarios utilicen clave única en ambas plataformas; y el portal es flexible.

El portal está implementado y se desarrolló para la Incubadora de Empresas de Alta Tecnología Genius. El URL para ingresar es http://www.geniusutec.com; y se espera que pueda ser una propuesta que en un futuro pueda ser implementada por muchas otras instituciones.

\section{Referencias}

Bilib (21 de 10 de 2015). "Gestion de Contenidos Web". Obtenido de

http://www.bilib.es/uploads/media/estudio_sistemas_ gestion_contenidos_web_cms.pdf

Carleto, J.A. (16 de 10 de 2015). "Claroline, una herramienta libre". Obtenido de http://jornadaie.unvm.edu.ar/ponencia12.pdf

Clarenc, C.A. (2013). 19 Plataformas de E-learning. Buenos Aires: Grupo Geipite.

E-ABC (15 de 09 de 2015). "E-AbcLearning". Obtenido de http://www.e-abclearning.com/queesunaplataformadeelearning

EducAr (10 de 09 de 2015). "Ministerio de Educación Argentina". Obtenido de

http://coleccion.educ.ar/coleccion/CD26/datos/ recursos/plataformas_virtuales.pdf

Faver, H. (20 de 09 de 2015). "Educación del futuro". Obtenido de http://www.listindiario.com/la-vida/2015/08/25/385434/ la-educacion-del-futuro

Moreira, M.A. (20 de 09 de 2015). "Tecedu Web". Obtenido de http://tecedu.webs.ull.es/textos/eLearning.pdf

Ntic (15 de 09 de 2015). "La Nave Tic". Obtenido de http://www.lanavetic.com/las-mejores-plataformas-elearning-de-software-libre/

Parra, C.L. (20 de 09 de 2015). "McGraw-Hill Interamericana de España". Obtenido de https://www.mhe.es/secundaria/informatica/8448168933/ archivos/Software/Ud07/ud_7_cms-Iljornadas.pdf

TES, N.O. (14 de 10 de 2015). "Plataformas de e-learning". Obtenido de http://plataformas-e-learning.wikispaces. com/TIPOS+DE+PLATAFORMAS

UNAM, U.N. (18 de 10 de 2015). "Computo Académico, UNAM". Obtenido de http://www.ru.tic.unam. mx:8080/bitstream/DGTIC/81750/1/mod4.pdf

Wise. (10 de 09 de 2015). “Cumbre mundial para la innovación en educacion". Obtenido de http://www.wise-qatar. org/ 